\title{
Erratum: Off-Shell Higgs Probe of Naturalness [Phys. Rev. Lett. 120, 111801 (2018)]
}

\author{
Dorival Gonçalves, Tao Han, and Satyanarayan Mukhopadhyay
}

(Received 3 July 2018; published 17 August 2018)

DOI: 10.1103/PhysRevLett.121.079902

Subsequent to our recent publication, we have noted an inadvertent numerical error in the computation of the corrections from the singlet scalar sector to the off-shell Higgs production process. The corrected differential distributions of the fourlepton invariant mass observable are presented in Fig. 1 (left). In view of this correction, the sensitivity reach at the 14 and $27 \mathrm{TeV}$ LHC runs are also modified by a small margin, as seen in Fig. 1 (right). Our primary conclusions remain unaltered and we summarize them as follows.

(1) The $2 \sigma(5 \sigma)$ reach at the $27 \mathrm{TeV}$ LHC using the off-shell probe is $m_{S} \sim 120 \mathrm{GeV}(100 \mathrm{GeV})$, for values of the portal coupling dictated by the naturalness relation.

(2) The off-shell analysis is found to have better sensitivity compared to realistic projections for the singlet pair production in the vector-boson fusion (VBF) channel, over the entire singlet mass range of interest $m_{S}>m_{h} / 2$.

One additional interesting feature observed is that there is an enhancement of sensitivity of the off-shell channel for values of $m_{S}$ close to $m_{t}$. This is because of the opening of two different thresholds close to each other, namely, the $2 m_{t}$ threshold in the triangle and box diagrams for $Z Z^{*}$ production and the $2 m_{S}$ threshold in the radiative correction from the scalar singlet to the same process.

In view of the revised differential distributions, we note in passing that for a typical parameter point with $5 \sigma$ reach at the $14 \mathrm{TeV}$ HL-LHC, e.g., $m_{S}=70 \mathrm{GeV}$ and $\lambda_{S}\left(m_{h}^{2}\right)=4.5$, we obtain around 425 signal events for $180<m_{4 \ell}<200 \mathrm{GeV}$, while the standard model (SM) background ( $q \bar{q}$ and $g g$ induced) event number is around 14800.
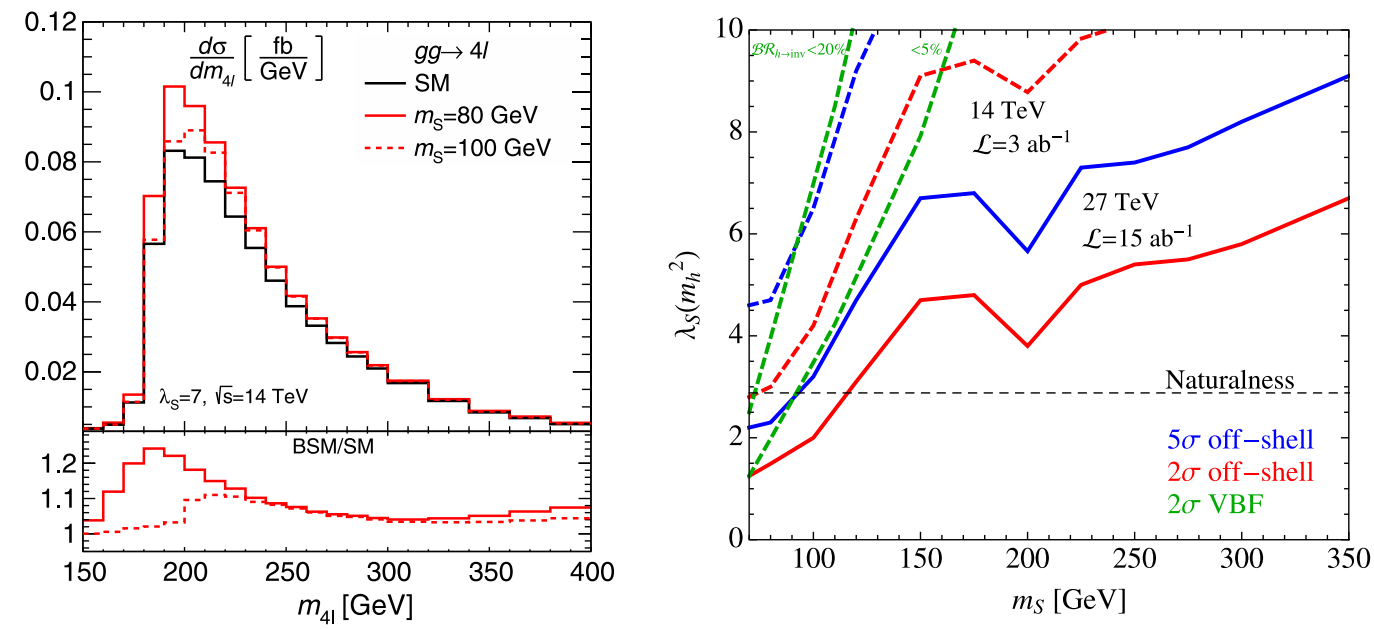

FIG. 1. Left: Four-lepton invariant mass distribution for the $g g \rightarrow 4 \ell$ process at the $14 \mathrm{TeV}$ LHC: in the SM (black line) and in the presence of an additional gauge singlet scalar (red line), including the one-loop electroweak effects from the singlet scalar sector. We show the signal ratio between the scalar singlet model and the SM in the bottom panel. Right: $2 \sigma$ (red line) and $5 \sigma$ (blue line) sensitivity on the singlet-Higgs coupling $\lambda_{S}$ at the scale $m_{h}^{2}$ as a function of the singlet scalar mass $m_{S}$ from the off-shell Higgs analysis at the $14 \mathrm{TeV}$ LHC with $\mathcal{L}=3 \mathrm{ab}^{-1}$ (dashed line) and at the $27 \mathrm{TeV}$ LHC with $\mathcal{L}=15 \mathrm{ab}^{-1}$ (solid line). For comparison, we also show the reach from the weak-boson fusion production of Higgs above its threshold, assuming the high-luminosity LHC $2 \sigma$ confidence level projections of $\mathcal{B R}(h \rightarrow$ invisible) $<20 \%$ (green dotted line) and 5\% (green dashed line), which correspond to a realistic projection and an idealistic limit of the systematic uncertainties on the background prediction, respectively.

Published by the American Physical Society under the terms of the Creative Commons Attribution 4.0 International license. Further distribution of this work must maintain attribution to the author(s) and the published articles title, journal citation, and DOI. 